Albelda Raga, José.

Profesor titular, UPV.

de Frutos, Estela.

Técnico de investigación, UPV.

Sgaramella, Chiara.

Doctoranda, UPV.

\title{
ARTE, NATURALEZA Y CONTEMPLACIÓN. Una propuesta teórico-experiencial a partir de las jornadas "La mística del cuerpo consciente".
}

\author{
TIPO DE TRABAJO \\ Comunicación.
}

PALABRAS CLAVE

Arte contemporáneo, gestión cultural, naturaleza, contemplación, atención consciente, espiritualidad.

KEY WORDS

Contemporary art, cultural management, nature, contemplation, mindfulness, spirituality.

RESUMEN

La presente comunicación surge del proyecto cultural La mística del cuerpo consciente, fruto de la colaboración entre el Centro de Investigación Arte y Entorno y el Laboratorio de Creaciones Intermedia, con el patrocinio de la UPV. La propuesta corresponde a un modelo de investigación que incorpora la gestión cultural para involucrar al público en una participación activa y experiencial. Partiendo de la actualización de las prácticas contemplativas tradicionales mediante un diálogo con el pensamiento ecológico y la creación contemporánea, el proyecto reivindica la experiencia de la meditación desde un prisma intercultural y laico, para explorar otros modos de conocimiento basados en la vivencia y la atención consciente. Las diversas actividades incluyen un amplio abanico de propuestas desde las más clásicas hasta las nuevas tecnologías, pasando por el walking art y otras fórmulas experienciales. Todas ellas se desarrollaron alrededor del tema del cuerpo como núcleo de conexión entre contemplación, naturaleza y arte, tomándolo como punto de partida para abordar cuestiones como la identidad, la empatía o la interdependencia.

\section{ABSTRACT}

This paper arises from the cultural project entitled La mistica del cuerpo consciente organized in collaboration with the Centre for Research on Art and Environment (CIAE) and the Intermedia Creations Lab (LCI) with the support of the Polytechnic University of Valencia. The proposal belongs to a research model that incorporates cultural management to encourage the active and experiential participation of the public. Starting with the update of traditional contemplative practices through a dialogue with ecological thinking and contemporary creation, the project reclaims the experience of meditation from an intercultural and secular point of view in order to explore other modes of knowledge based on experience and mindfulness. The proposal included a wide range of activities from classical art forms to new media, walking art and other experiential practices. All of them revolved around the body, conceived as the connection point among contemplation, nature and art and as a starting point to explore the issues of identity, empathy and interdependence. 


\section{CONTENIDO}

\section{INTRODUCCIÓN}

El presente estudio parte de las Jornadas de arte, naturaleza y contemplación, "La mística del cuerpo consciente", iniciativa innovadora en el ámbito de la educación y la gestión cultural, y propone una reflexión crítica acerca de la relación entre cultura, creación artística y experiencia contemplativa. Ideado por Estela de Frutos, Chiara Sgaramella y José Albelda, el proyecto se realizó en Octubre de 2014 gracias a al apoyo de la Universidad Politécnica de Valencia y a la colaboración entre el Centro de Investigación Arte y Entorno y el Laboratorio de Creaciones Intermedia. La propuesta plantea un modelo de investigación teórico-práctico enraizado en los retos de la sociedad actual, englobando un programa de gestión cultural dirigido a implicar al público en una participación activa y experiencial. En el contexto de la crisis ecológica actual, estas jornadas responden a la necesidad de vincular diferentes campos del saber en la construcción de paradigmas culturales más sostenibles, que refuercen el proceso de tránsito hacia una sociedad ecólogica. En concreto, frente a la visión dicotómica de la relación cultura-naturaleza ${ }^{1}$ que caracteriza el modelo de desarrollo dominante, la iniciativa aspira a propiciar una interacción responsable con la biosfera a través del cultivo de una conciencia individual expandida y en estrecho diálogo con el ecosistema. Basándose en las ideas enunciadas por Félix Guattari en Las tres ecologías ${ }^{2}$, el proyecto pretende integrar las dimensiones psico-corporal, socio-cultural y medioambiental en un continuum orgánico conformado por relaciones interdependientes. Para abordar tal complejidad, se decidió apostar por un enfoque interdisciplinar y teórico-experiencial.

En la definición de los ejes temáticos de la propuesta, se adoptó el término "contemplación" para analizar la meditación desde una perspectiva laica e intercultural. Si bien el interés por reencontrar una dimensión interior o "espiritual" del ser humano es creciente, hay cierta polémica lógica sobre el uso del término "espiritualidad", por las múltiples connotaciones que lleva implícita desde las diferentes tradiciones espirituales ${ }^{3}$. A partir de este planteamiento general, se elaboró un programa cultural amplio y diverso que ponía en relación el pensamiento ecológico con la creación artística y con el potencial transformador de las prácticas contemplativas. Este gran abanico de actividades incluía conferencias de tipo teórico y fórmulas educativas más innovadoras y participativas como talleres experienciales de meditación y conciencia corporal en contacto con la naturaleza. Desde el punto de vista artístico, se intentó visibilizar formas de arte contemporáneo vinculadas a la contemplación y a una visión holística y respetuosa del medioambiente, partiendo de propuestas tradicionales -como la pintura ${ }^{4}$ - hasta abarcar géneros mas experimentales como la performance, la videocreación, el arte sonoro y la arquitectura sostenible.

\section{DESARROLLO}

\section{Cuerpo e identidad: hacia una cosmovisión de interdependencia}

Las actividades desarrolladas giraron alrededor del cuerpo, como punto de encuentro entre las distintas disciplinas incluidas en el proyecto cultural. Este tema se enfocó desde diferentes puntos de vista: el cuerpo entendido como lugar de numerosas deconstrucciones y discursos de identidad desde el arte contemporáneo; como fundamento de la atención plena en las prácticas contemplativas, y finalmente como parte integrante de la biosfera desde el punto de vista del pensamiento ecológico. En las jornadas, esta mirada múltiple y a la vez integradora fue acompañada por una recuperación del cuerpo en el proceso de acercamiento ecosistémico a la contemplación. En efecto, frente a la tradición judeo-cristiana de una mística entendida como huida de la corporeidad en busca de una dimensión trascendente, se propuso una investigación experiencial sobre la práxis contemplativa, dedicando especial atención al papel de los sentidos. Dicho planteamiento enlaza con algunos aspectos del budismo zen, en el que el cuerpo es un elemento clave para el desarrollo de la conciencia a través de la conexión con el presente. Además, en la tradición zen, el cuerpo humano no se considera como una entidad separada de su entorno. En la conferencia titulada Construcción y deconstrucción de la autoimagen corporal, el maestro de la escuela Soto Zen Dokushô Villalba explicó cómo, según las antiguas enseñanzas budistas, el cuerpo se extiende más allá de los límites que normalmente se le atribuyen. Mediante un análisis crítico de las representaciones convencionales del cuerpo, Villalba destacó la importancia de entender la inconsistencia en la que se basa la actual concepción del cuerpo como algo separado del resto, poniendo énfasis en su conexión con todo lo demás ${ }^{5}$. Esta visión amplia de la identidad basada

\footnotetext{
${ }^{1}$ Una exposición completa acerca de la dialéctica cultura/naturaleza puede encontrarse en Albelda, J. y Saborit, J., La construcción de la Naturaleza, Generalitat Valenciana, Valencia, 1997.

${ }^{2}$ Guattari, Félix, Las tres ecologías, Pre-Textos, Valencia, 1996.

${ }^{3}$ Una interesante reflexión acerca del término "espiritualidad" aparece en Martinez Lozano, E.; Vida en plenitud, apuntes para una espiritualidad transreligiosa, PPC, 2012.

${ }^{4}$ Entre los artistas invitados Domenec Corbella, pintor y profesor en la Universidad de Barcelona, ofreció una videoconferencia titulada El espíritu en la pintura, ilustrando la relación entre naturaleza y espiritualidad en su trayectoria personal; (Corbella, D. (Ed.); El espíritu en la creatividad artística, Promociones y Publicaciones Universitarias, Barcelona, 2010).

${ }^{5}$ El maestro zen japonés Eihei Dôgen en su obra titulada Shôbôgenzô ("La Preciosa Visión del Dharma Verdadero"), afirma:

"El universo entero que se extiende en las diez direcciones / no es más que el ojo de este monje.

El universo entero que se extiende en las diez direcciones / no es más que la palabra cotidiana de este monje.

El universo entero que se extiende en las diez direcciones / no es más que el cuerpo de este monje." (Villalba, D.; Kômyô.Clara Luz, Miraguano Ediciones, Madrid, 2010).
} 
en la interdependencia entre ser humano y ecosistema resulta particularmente significativa y presenta numerosos puntos de contacto con los fundamentos éticos del pensamiento ecológico contemporáneo.

En la cultura occidental, la experiencia del cuerpo en comunión con el entorno vinculada a una incipiente ética ambiental se ve reflejada en el paseo contemplativo de Thoreau ${ }^{6}$, en el que se funden la serenidad derivada de la observación de la naturaleza y la defensa de la vida y de la libertad tanto de los humanos como de las otras especies. La vivencia y el sentido metafórico del camino han inspirado también el quehacer artístico de Pep Mata -walking artist catalán- que, en la línea de Richard Long y Hamish Fulton, documenta mediante el uso de la fotografía su paso por lugares ásperos y solitarios, como las rutas de alta montaña del Pirineo. En la ponencia ofrecida en las jornadas, Mata describió su proceso creativo como un ritual de transformación interior que surge a partir del tránsito y del contacto con el paisaje. Para el artista el caminar se convierte en una acción meditativa en la que el ritmo de la respiración, la cadencia repetitiva de los pasos y el movimiento físico permiten tranquilizar la mente y abrir el llamado "ojo interior"7. Una vez alcanzado este espacio de silencio, se manifiesta la experiencia del tiempo presente y se hace accesible una lectura distinta y más profunda de la realidad. Este particular estado de consciencia, según Mata, genera una experiencia de unidad con la naturaleza en la que es posible percibir las dimensiones intangibles del territorio. La obra de arte se configura como registro poético de este proceso contemplativo, una estrategia para capturar y compartir el asombro y las emociones despertadas por los lugares explorados. Las series fotográficas de Pep Mata son, en efecto, una invitación a detenerse en silencio para observar los escenarios naturales en su poderosa y a la vez frágil belleza.
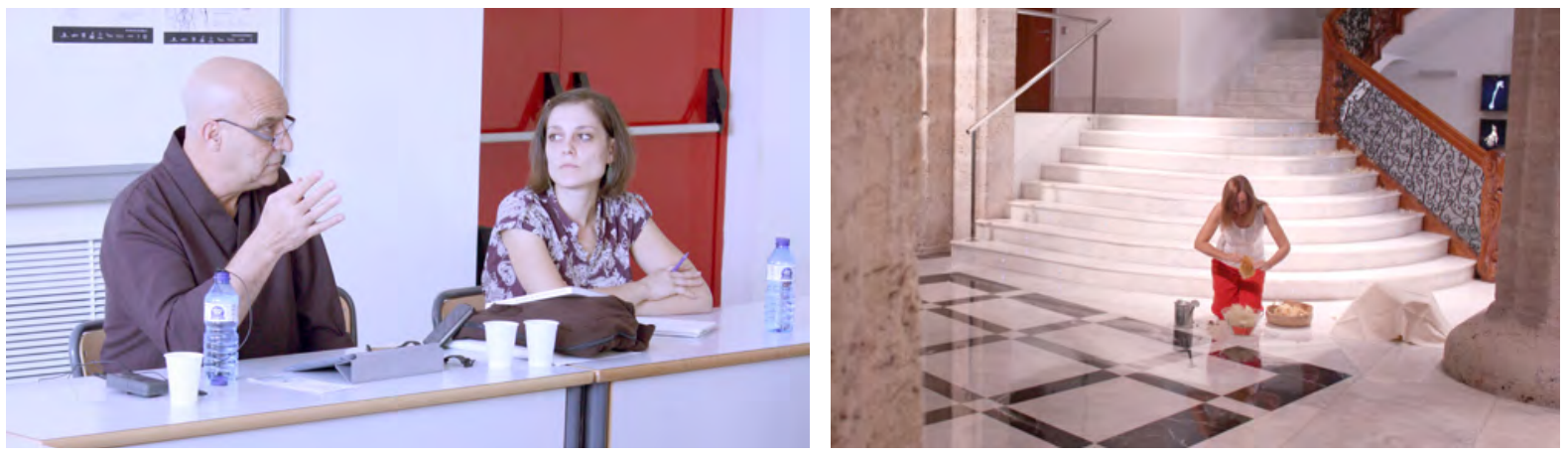

Imagen 1. Conferencia del maestro zen Dokushô Villalba. Imagen 2. B. Codonyer, Las flores silvestres de mi jardín, 2014.

El concepto de fragilidad es recurrente también en la práctica artística de Beatriu Codonyer. La creadora valenciana indaga principalmente el territorio de la performance, donde arte y vida se configuran como una unidad indisociable y el cuerpo representa el centro de la experiencia creativa. Su trabajo se caracteriza por la esencialidad y por la carga simbólica de los elementos naturales agua, ceniza, huevos y fragmentos vegetales- que emplea en la realización de sus piezas. Invitada a participar en las jornadas con una propuesta inédita, la performer ideó una acción íntima y sutil titulada Las flores silvestres de mi jardín, en la que intentaba revitalizar inflorescencias secas sumergiéndolas en un cuenco de cristal lleno de agua. El ritmo dilatado y la reiteración de los movimientos generaban una atmósfera de reflexión, enfatizando el significado metafórico de la obra y a la vez planteando la acción creativa no sólo como la concreción plástica de una idea, sino también como un estímulo para resignificar la relación con el entorno vital.

\section{Arte, contemplación y tecnología en tiempos de crisis ecológica global}

Uno de los aspectos significativos del proyecto cultural La mística del cuerpo consciente fue la inclusión de las nuevas tecnologías al servicio de la cultura, la sostenibilidad y el desarrollo humano. El papel de la tecnología en la sociedad actual es un tema amplísimo y su estudio excede los límites del presente artículo. Sin embargo, creemos que es importante apuntar algunas cuestiones acerca de su utilización desde el pensamiento ecológico y en relación a la contemplación. Con frecuencia las reivindicaciones de los movimientos vinculados a la ética ambiental son asociadas a ideologías teñidas de primitivismo y contrarias a los avances tecnocientíficos. No obstante, en este proyecto cultural, lejos de defender posturas anti-tecnológicas, se consideró el uso de las nuevas tecnologías (video, internet, etc.) como una preciosa herramienta de innovación artística y sensibilización ecológica. Efectivamente, entre sus múltiples ventajas, la tecnología genera nuevos recursos estéticos para todas las disciplinas artísticas y, gracias a la difusión de internet, permite a un público más numeroso y diverso acceder a contenidos culturales alternativos a la visión capitalista

\footnotetext{
${ }^{6}$ Thoreau, H. D.; Caminar, Ardora Ediciones, Madrid, 1998.

${ }^{7}$ Desde la antigüedad muchos autores se han referido metafóricamente a este "ojo del espíritu" o "tercer ojo" como una nueva perspectiva de conocimiento frente a los dos primeros que serían el "ojo de la carne" y el "ojo de la razón". Uno de los autores que ha retomado este tema en la actualidad tratando de establecer puentes entre oriente y occidente es Ken Wilber (Wilber, K.; Los tres ojos del conocimiento, Kairós, Barcelona, 1991 y El ojo del espíritu, Kairós, Barcelona, 1998).
} 
dominante. Por lo tanto, no representa sólo un instrumento de homogeneización, de desconexión y huida hacia lo virtual, sino que puede ser utilizada de manera eficaz para incidir constructivamente en la realidad contemporánea.

Sin embargo, es imprescindible señalar también los posibles peligros vinculados a un uso acrítico de las tecnologías, como la fe irracional en una tecno-ciencia omnipotente y omnipresente en quien se delega la futura solución de todos los problemas del presente, incluidos los problemas ecológicos o la ilusión de poder escapar a la finitud de la condición humana ${ }^{8}$. Asimismo, la intromisión tecnológica en todos los aspectos de la vida junto al exceso de información y estímulos generan dispersión, eliminando de facto los espacios de introspección y reflexión. Para evitar algunos de estos inconvenientes, se decidió integrar las nuevas tecnologías junto con otras fórmulas que fomentaran la conciencia corporal, la presencia y la experiencia directa del entorno. Este enfoque supone en sí mismo una reformulación no sólo de la actual invasión tecnológica que estamos viviendo, sino también de las habituales estereotipos asociados a la meditación. La conjunción de ambos en el ámbito de las jornadas, se concretó en una instalación efímera realizada en los jardines de la UPV y construida con materiales de bajo impacto ambiental. En ella se integró una propuesta de arte sonoro electrónico a cargo de los artistas Deco Nascimento y Carlos García Miragall, con la colaboración del Laboratorio de Creaciones Intermedia. Esta intervención permitió crear un lugar de contemplación en diálogo con la creación contemporánea y el espacio natural.

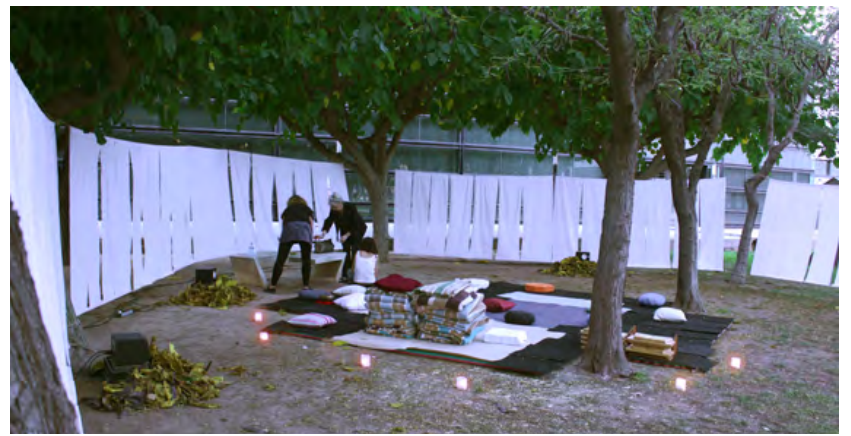

Imagen 3. Instalación efímera en los jardines de la UPV.

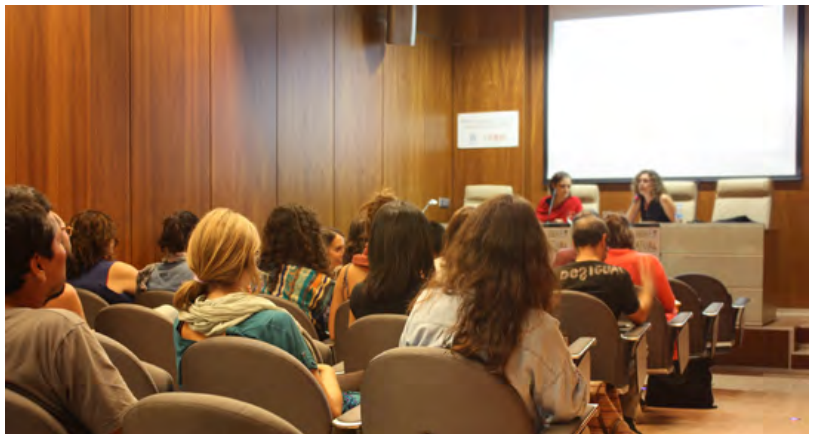

Imagen 4. Inner Nature, muestra de videoarte. Valencia, 2014.

Otra experiencia significativa fue la muestra internacional de videoarte Inner Nature, organizada en colaboración con Lorena Rodriguez Mattalía, experta en arte videográfico y docente de la UPV. En la exposición participaron artistas invitados y creadores seleccionados mediante una convocatoria pública en la que se podían presentar videocreaciones inspiradas en la temática mencionada. Los videos admitidos se mostraron en diferentes eventos expositivos en Valencia, Salamanca y en Jyväskylä (Finlandia), además la itinerancia del proyecto prevé dos otras etapas en Marnay sur Seine (Francia) y en Santiago de Chile. La muestra comprendía distintos acercamientos a la experiencia contemplativa de la naturaleza y una interesante variedad de resoluciones estéticas. Especialmente revelador fue el uso del dispositivo videográfico como estrategia para profundizar en la práctica de la atención consciente mediante un peculiar manejo del tiempo, del silencio y del vacío, en antítesis con el lenguaje mediático contemporáneo, generalmente caracterizado por la saturación sensorial. Además, el formato digital de las obras y la creación de una plataforma online facilitaron la difusión del proyecto, minimizando su impacto económico y ambiental. Inner Nature demostró ser una fórmula idónea de gestión cultural, susceptible de ser ampliada en próximas ediciones y aplicable a múltiples contextos.

\section{El papel de la experiencia: talleres de conciencia y creatividad}

Entre los objetivos principales de las jornadas La mística del cuerpo consciente figuraba la recuperación del valor de lo experiencial en la educación, en concreto la experimentación artística y la creación de un vínculo consciente con el entorno social y natural. La relación intrínseca entre experiencia, vida y conocimiento no es un hallazgo reciente. En efecto, se trata de un concepto presente en diferentes tradiciones espirituales así como en la pedagogía ${ }^{9}$ y a la vez en los movimientos vanguardistas y neovanguardistas del arte occidental. Sin embargo, el paradigma científico-racionalista actualmente dominante en todos los ámbitos de la vida, tiende a potenciar las habilidades intelectuales en detrimento de otros recursos cognitivos vinculados a la experiencia como la inteligencia emocional, la creatividad, la intuición o la consciencia corporal. Con el fin de equilibrar este enfoque, se incluyeron en el programa de las jornadas actividades poco habituales en un contexto académico para fomentar un modelo de aprendizaje vivencial.

\footnotetext{
${ }^{8}$ El filósofo Jorge Riechmann analiza los distintos movimientos de huida vinculados a la fe irracional en las tecnologías clasificándolos en: "huida de los límites al crecimiento económico: nuevos caminos para intentar proseguir la expansión, por ejemplo con nuevas fuentes de energía (fusión nuclear) y desafíos para la naturaleza entrópica de nuestro mundo (nanotecnologías). Huida del planeta Tierra: la fuga al cosmos. Huida de la naturaleza humana: creación de "post-humanos" mediante ingeniería genética y simbiosis hombre-máquina. Huida de la sociedad hacia el ciberespacio" (Riechmann, J.; Gente que no quiere viajar a Marte, Libros de la Catarata, Madrid, 2004, p. 36).

${ }_{9}^{9}$ Dewey, J.; Experiencia y educación, Biblioteca Nueva, Madrid, 2004.
} 
En primer lugar, se organizó una sesión abierta de meditación en colaboración con el Centro Budista de Valencia, para cultivar la consciencia corporal y la atención plena mediante antiguas técnicas de respiración como el Anapanasati. Además, se realizaron talleres experienciales dirigidos por Gema Hoyas y José Abelda en los que se compaginaba la escucha y exploración del cuerpo con la contemplación de la naturaleza a través de los sentidos para desencadenar procesos creativos y autoexpresivos. Sucesivamente, se incorporaron también prácticas dirigidas a generar un vínculo físico y sensitivo con los elementos naturales del contexto y así establecer una relación de empatía entre el yo humano y el ecosistema. En efecto, la consciencia de pertenecer a un todo, siendo partícipes del mismo espacio-tiempo y de un proceso evolutivo común, representa un elemento clave para promover una actitud de respeto ante la naturaleza.
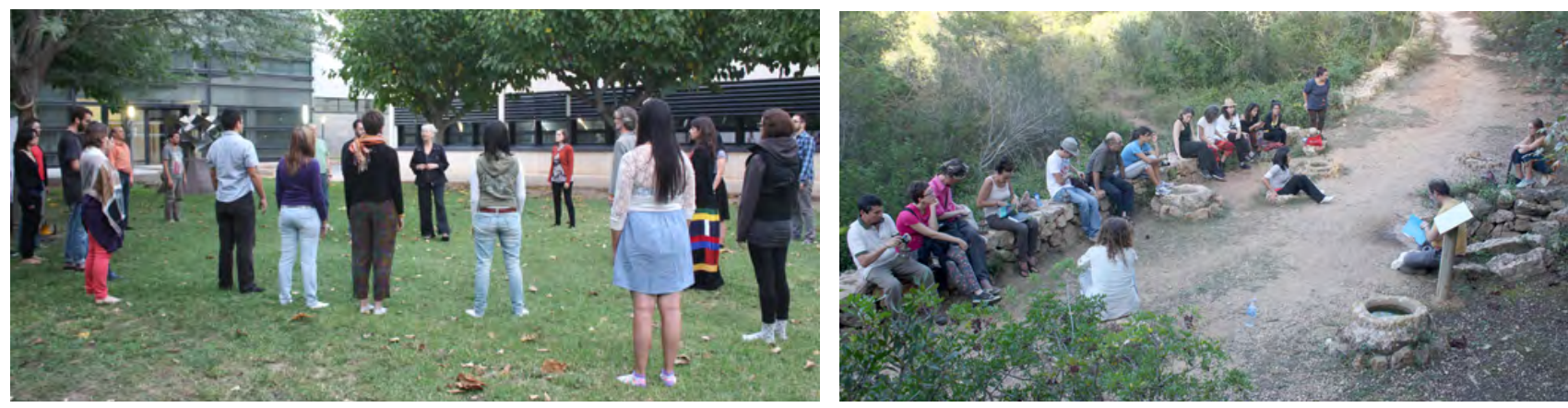

Imagen 5. Talleres experienciales

Imagen 6. Workshop con el artista Josep Ginestar en Gata de Gorgos

Por último, como culminación del proyecto cultural, se ofreció un workshop con el artista Josep Pedrós i Ginestar, cuya obra reúne poesía visual, fascinación por el paisaje y la espiritualidad sufi, vertiente mística del Islam. El taller se llevó a cabo en un paraje natural de la localidad de Gata de Gorgos (Alicante) donde, bajo la guía del artista, los participantes disfrutaron de una experiencia de contemplación más profunda a partir de la inmersión y del contacto no mediado con el entorno natural. Asimismo, pudieron enriquecer su sensibilidad artística, experimentando libremente con diferentes lenguajes plásticos e incorporando en su proceso creativo la vivencia física y espiritual del paisaje.

\section{CONCLUSIONES}

La experiencia de La mística del cuerpo consciente ha representado un fructífero encuentro entre disciplinas en apariencia distantes alrededor de la experimentación de estrategias culturales viables frente a la crisis ecológica que vivimos. La iniciativa fue el resultado del trabajo en red de distintas instituciones y entidades, un eficaz ejemplo de colaboración ante este reto común.

La intención de influir constructivamente en los procesos de transición hacia la sostenibilidad fue reforzada por la adopción de un enfoque teórico-practico de investigación y la inclusión de propuestas didácticas y de gestión cultural que permitieran una implicación participativa del público. En concreto, el acercamiento desde el cuerpo a las prácticas de contemplación en diálogo con la ecología, se reveló como una de las posibles vías para superar la escisión cultura/naturaleza y cultivar una conciencia sensible a los valores de respeto y cuidado del ecosistema. En este sentido, de acuerdo con el pensamiento de Guattari, las jornadas han constituido una contribución a un cambio de cosmovisión que no concierne solo "a las relaciones de fuerza visibles a gran escala, sino tambien a los campos moleculares de sensibilidad, de inteligencia y de deseo" ${ }^{10}$.

Desde el punto de vista teórico, las conferencias y las actividades experienciales permitieron realizar una amplia reflexión interdisciplinar acerca de cuestiones como la identidad, la empatía y la interdependencia desde perspectivas diversas y enriquecedoras. Un aspecto innovador del proyecto fue la valorización del papel de las nuevas tecnologías revisitando las tradiciones contemplativas en conexión con el arte contemporáneo. En lugar de apoyar estereotipos anti-tecnológico, se apostó por un uso responsable y creativo de las nuevas tecnologías (video, internet, etc.) al servicio del desarrollo humano y de la sostenibilidad.

El interés despertado por este interesante modelo de gestión cultural ha abierto el camino a nuevas perspectivas de continuidad y a la introducción de elementos de innovación en ámbito académico. En efecto, en 2015 se llevará a cabo la segunda edición de la muestra de videoarte Inner Nature Exhibition y se propondrá la creación de un aula de meditación en la Universidad Politécnica de Valencia.

\footnotetext{
${ }^{10}$ Guattari, Félix, Las tres ecologías, Pre-Textos, Valencia, 1996, p. 10.
} 


\section{FUENTES REFERENCIALES}

ALBELDA, J. y SABORIT, J.; La construcción de la Naturaleza, Generalitat Valenciana, Valencia, 1997.

CORBELLA, D. (Ed.); El espíritu en la creatividad artística, Promociones y Publicaciones Universitarias, Barcelona, 2010.

DEWEY, J.; Experiencia y educación, Biblioteca Nueva, Madrid, 2004.

GUATTARI, F.; Las tres ecologías, Pre-Textos, Valencia, 1996.

MATA, P.; Andar-i-ego. El trazador de caminos y direcciones, Ediciones Saragossa, Universidad de Barcelona, Barcelona, 2013.

MARTÍNEZ LOZANO, E.; Vida en plenitud, apuntes para una espiritualidad transreligiosa, PPC, 2012.

RIECHMANN, J.; Gente que no quiere viajar a Marte, Libros de la Catarata, Madrid, 2004.

THOREAU, H. D.; Caminar, Ardora Ediciones, Madrid, 1998.

VILLALBA, D.; Kômyô. Clara Luz, Miraguano Ediciones, Madrid, 2010.

WILBER, K.; El ojo del espíritu, Kairós, Barcelona, 1998.

WILBER, K.; Los tres ojos del conocimiento, Kairós, Barcelona 1991

\section{PÁGINAS WEB}

Codonyer, B.: https://vimeo.com/user18715201

Mata, P.: www.pepmata.com

Villalba, D.: www.dokushovillalba.blogspot.com.es 